\title{
ABOUT STABILITY ESTIMATES AND RESOLVENT CONDITIONS
}

\author{
J. L. M. VAN DORSSELAER, J. F. B. M. KRAAIJEVANGER \\ and M. N. SPIJKER \\ Department of Mathematics and Computer Science, University of Leiden \\ Niels Bohrweg 1, 2333 CA Leiden, The Netherlands \\ E-mail: SPIJKER@RULCRI.LEIDENUNIV.NL
}

\section{Introduction}

1.1. Power boundedness and resolvent conditions. A central issue in the stability analysis of numerical methods is the question whether given matrices have powers that are uniformly bounded. Therefore, we consider for an arbitrary $s \times s$ matrix $B$ the stability property

$$
\left\|B^{n}\right\| \leq M_{0} \quad \text { for } n=0,1,2, \ldots,
$$

where $M_{0}$ is a positive constant. For the time being $\|\cdot\|$ stands for the spectral norm (i.e. the matrix norm induced by the Euclidean norm on $\mathbb{C}^{s}$ ).

The famous Kreiss matrix theorem (see e.g. [11], [32]) relates (1.1) to conditions on $B$ which are easier to verify than (1.1). One of these conditions involves the so-called resolvent $(\zeta I-B)^{-1}$ of $B$, and reads as follows:

(1.2) $\quad \zeta I-B$ is invertible and $\left\|(\zeta I-B)^{-1}\right\| \leq M_{1}(|\zeta|-1)^{-1}$ for all complex numbers $\zeta \notin D$.

Here $M_{1}$ is a positive constant, $I$ the $s \times s$ identity matrix and $D=\{\zeta: \zeta \in \mathbb{C}$ and $|\zeta| \leq 1\}$ the closed unit disk.

1991 Mathematics Subject Classification: 65M12, 39A11, 65L20.

The research of the first author has been supported by the Netherlands Organization for Scientific Research (N.W.O.).

The research of the second author has been made possible by a fellowship of the Royal Netherlands Academy of Arts and Sciences (K.N.A.W.).

Correspondence should be addressed to the third author.

The paper is in final form and no version of it will be published elsewhere. 
If (1.1) is satisfied, then the spectrum of $B$ lies in $D$, so that for all $\zeta \notin D$ the matrix $\zeta I-B$ is invertible and

$$
\left\|(\zeta I-B)^{-1}\right\|=\left\|\sum_{k=0}^{\infty} \zeta^{-k-1} B^{k}\right\| \leq \sum_{k=0}^{\infty}|\zeta|^{-k-1} M_{0}=M_{0}(|\zeta|-1)^{-1} .
$$

Hence (1.1) implies (1.2) with $M_{1}=M_{0}$. The Kreiss matrix theorem asserts that, conversely, (1.2) implies (1.1) with $M_{0}$ depending only on $M_{1}$ and the dimension $s$, but otherwise independent of the matrix $B$.

The Kreiss theorem has often been used in the stability analysis of numerical methods for solving initial value problems for partial differential equations. In the classical situation the matrices $B$ are obtained by Fourier transformation of the numerical solution operators, and they stand essentially for the so-called amplification matrices (see e.g. [32]). These matrices are of a fixed finite order $s$. On the other hand, the implication of (1.1) by (1.2) can also be used without Fourier transformation, with $B$ standing for the numerical solution operators themselves. In this situation we are dealing with a family of matrices $B$ of finite - but not uniformly bounded - orders $s$. Therefore, of particular interest is the dependence of the stability constant $M_{0}$ in (1.1) on the dimension $s$.

Various authors [25], [24], [38], [19], [37] studied the size of (the optimal) $M_{0}$ as a function of $M_{1}$ and $s$, and recently some open problems in this field were solved. Moreover, the implication of (1.1) by (1.2) as discussed above has recently been generalized in several directions. More general norms than the spectral norm have been dealt with and the resolvent condition (1.2) has been adapted to domains different from the unit disk $D$. In the latter case the matrices $B$ in (1.1) and (1.2) are not the same, but are related to each other by a rational transformation.

1.2. Scope of the paper. In this paper we review some of the recent results mentioned above. Moreover, we announce some generalizations and improvements, the full proof of which will be given in a forthcoming paper by the authors.

In Section 2 we still deal with the resolvent condition (1.2) with respect to the unit disk $D$. But, we consider general norms on the vector space of all $s \times s$ matrices. In this situation we focus on the best upper bounds for $\left\|B^{n}\right\|$ that are possible under condition (1.2).

In Section 3 we relate estimates like (1.1) more explicitly to the stability analysis of numerical methods for the solution of initial-boundary value problems in partial differential equations. We show that in this analysis it is useful to consider resolvent conditions with respect to regions $V \subset \mathbb{C}$ that are different from the unit disk $D$. Moreover, in Subsection 3.1 we touch on the point that in order to check estimates like (1.1) it is, in general, totally unsatisfactory to study only the eigenvalues of $B$ and to require e.g. that they are all contained in the interior of the unit disk $D$. In Subsection 3.2 we give a review of stability estimates from the literature based on resolvent conditions with respect to general regions $V$. Subsection 3.3 provides various comments on these estimates. 
In this paper we focus on stability estimates for the so-called one-step methods. For stability considerations not reviewed in our paper, but still closely related, we refer to our extensive list of references. In particular, for (linear) multi-step methods see [5], [7], [20], [21], [30], [31].

\section{Stability estimates under resolvent conditions with respect to the unit disk}

2.1. The classical situation for arbitrary $M_{1} \geq 1$. As already mentioned in the introduction, the Kreiss matrix theorem asserts, for the spectral norm, that the resolvent condition (1.2) implies power boundedness (1.1) with a stability constant $M_{0}$ depending only on $M_{1}$ and the dimension $s$. According to Tadmor [38], Kreiss's original proof [11] yields an upper bound $\left\|B^{n}\right\| \leq M_{0}$ with

$$
M_{0} \simeq\left(M_{1}\right)^{s^{s}},
$$

which is far from sharp. After successive improvements by various authors (cf. [25], [24]), Tadmor was the first to prove a bound that is linear in $s$,

$$
\left\|B^{n}\right\| \leq 32 e \pi^{-1} s M_{1}
$$

(cf. [38]). LeVeque and Trefethen [19] lowered this upper bound to $2 e s M_{1}$, and conjectured that the latter bound can be improved further to

$$
\left\|B^{n}\right\| \leq e s M_{1} \quad \text { for } n=0,1,2, \ldots
$$

Moreover, these authors showed by means of a counterexample that the factor $e$ in (2.1) cannot be replaced by any smaller constant - if the upper bound is to be valid for arbitrary factors $M_{1}$ in (1.2) and arbitrarily large integers $s$.

Smith [35] proved a result which combined with the arguments in [19] leads to the improved upper bound $\left\|B^{n}\right\| \leq \pi^{-1}(\pi+2) e s M_{1}$, which is an improvement over the upper bound $2 e s M_{1}$, but still weaker than the conjecture (2.1). The conjecture was finally proved to be true in [37] (see also [40]).

In addition to the upper bound (2.1), which is linear in $s$ and independent of $n$, it is possible to derive an upper bound from (1.2), which is linear in $n$ and independent of $s$. By the Cauchy integral formula (see e.g. [4, p. 205]) we have

$$
B^{n}=\frac{1}{2 \pi i} \int_{\Gamma} \zeta^{n}(\zeta I-B)^{-1} d \zeta
$$

where the contour of integration $\Gamma$ is any positively oriented circle $|\zeta|=1+\varepsilon$ with $\varepsilon>0$. Choosing $\varepsilon=1 / n$ it readily follows from $(1.2),(2.2)$ that

$$
\left\|B^{n}\right\| \leq\left(1+\frac{1}{n}\right)^{n}(n+1) M_{1} \leq e(n+1) M_{1} \quad \text { for } n=1,2,3, \ldots
$$

(see also [17], [21], [30]).

In the next subsection we will discuss a generalization of the upper bounds (2.1), (2.3) to norms different from the spectral norm. We will also investigate the sharpness of these bounds in the general case. 
2.2. Stability estimates for arbitrary $M_{1} \geq 1$ and arbitrary norms. In this subsection we consider a generalization of the upper bounds (2.1), (2.3) to the case where $\|\cdot\|$ is an arbitrary norm on $\mathbb{C}^{s, s}$, the vector space of all complex $s \times s$ matrices. If the norm is submultiplicative (i.e., $\|A B\| \leq\|A\|\|B\|$ for all $A, B \in \mathbb{C}^{s, s}$ ) the norm is called a matrix norm. Norms for which $\|I\|=1$ are called unital (see e.g. [9]). $\mathbb{C}^{s, s}$.

Theorem 2.1. Let $s \geq 1, B \in \mathbb{C}^{s, s}$ and $\|\cdot\|$ denote an arbitrary norm on

(a) If (1.1) holds for some $M_{0}$, then (1.2) holds with $M_{1}=M_{0}$;

(b) If (1.2) holds for some $M_{1}$, then

$$
\left\|B^{n}\right\| \leq(1+1 / n)^{n} \min (s, n+1) M_{1} \quad \text { for } n=1,2,3, \ldots
$$

The proof of (a) is the same as the proof in Subsection 1.1 for the spectral norm. Since also the proof of (2.3) as given in Subsection 2.1 remains valid for arbitrary norms, the proof of (b) is complete if we can show

$$
\left\|B^{n}\right\| \leq(1+1 / n)^{n} s M_{1} \quad \text { for } n=1,2,3, \ldots
$$

Property (2.5) can be proved by using ideas from [19] and by using the HahnBanach theorem (cf. e.g. [34]) in a similar fashion as in [18]. The full proof of (2.5), as well as of all subsequent theorems stated below without proof, will be given in a forthcoming paper by the authors.

In the following theorem we focus on the sharpness of the bound (2.4) in case $n=s-1$.

TheOREM 2.2. Let $s \geq 2$ and an arbitrary norm $\|\cdot\|$ on $\mathbb{C}^{s, s}$ be given. Then

$$
\sup \left\{\left\|B^{s-1}\right\| / M_{1}(B): B \in \mathbb{C}^{s, s} \text { and } M_{1}(B)<\infty\right\}=\left(1+\frac{1}{s-1}\right)^{s-1} s,
$$

where $M_{1}(B)$ denotes the smallest $M_{1}$ such that (1.2) holds (we set $M_{1}(B)=\infty$ if (1.2) is not satisfied for any $\left.M_{1}\right)$.

Corollary 2.3. Let, for each $s \geq 1$, a norm $\|\cdot\|=\|\cdot\|^{(s)}$ be given on $\mathbb{C}^{s, s}$. Then there exist matrices $B_{s} \in \mathbb{C}^{s, s}$ for $s=1,2,3, \ldots$, such that $M_{1}\left(B_{s}\right)<\infty$ and

$$
\left\|\left(B_{s}\right)^{s-1}\right\|^{(s)} \sim e s M_{1}\left(B_{s}\right) \quad(\text { as } s \rightarrow \infty),
$$

where $M_{1}\left(B_{s}\right)$ has the same meaning as in Theorem 2.2 .

Proof. Immediate from Theorem 2.2.

The above corollary was proved in [19] for the spectral norm. Our proof of Theorem 2.2 is essentially based on ideas taken from that paper.

In view of (2.4) the estimate (2.1) is valid in the situation of general norms $\|\cdot\|$ on $\mathbb{C}^{s, s}$. By virtue of Corollary 2.3 this general version of $(2.1)$ is sharp in the sense of (2.7). However, it should be emphasized that this does not resolve the sharpness question for given fixed $M_{1}$, since $M_{1}\left(B_{s}\right)$ may become unbounded in 
(2.7) as $s \rightarrow \infty$. In the next two subsections we will focus on the situation where $M_{1}$ is a given fixed number.

2.3. About the best stability estimates for $M_{1}=1$. In the special situation where the resolvent condition (1.2) holds with $M_{1}=1$, the upper bound (2.4) can be improved in various ways. First we concentrate on arbitrary matrix norms on $\mathbb{C}^{s, s}$, and at the end of this subsection we focus on matrix norms $\|\cdot\|_{p}$ induced by the $p$ th Hölder norm on $\mathbb{C}^{s}($ for $p=1,2, \infty)$.

THEOREM 2.4. Let $s \geq 1, B \in \mathbb{C}^{s, s}$ and $\|\cdot\|$ denote an arbitrary matrix norm on $\mathbb{C}^{s, s}$. If (1.2) holds with $M_{1}=1$, then

$$
\left\|B^{n}\right\| \leq \sqrt{2 \pi(n+1)} \quad \text { for } n=0,1,2 \ldots
$$

Proof. From (1.2) with $M_{1}=1$ it can be seen that the matrix norm $\|\cdot\|$ is unital. Therefore $\mathbb{C}^{s, s}$ is a complex Banach algebra in the sense of $[2$, Sections 6 , 8], and we can apply [2, Theorem 16] so as to get $\left\|B^{n}\right\| \leq e^{n} n^{-n} n$ !. An application of Stirling's formula (see e.g. [1, formula 6.1.38] or [8, Section 8.5]) yields (2.8). Another proof can be given e.g. along the lines of [21, Theorem 2.1].

As the next theorem shows, the growth with $\sqrt{n}$ in (2.8) is not unnecessarily pessimistic. The proof of the theorem is based on arguments from [21] and [16].

THEOREM 2.5. There exists a constant $C>0$ such that

$$
\sup _{s,\|\cdot\|, B}\left\|B^{n}\right\| \geq C \sqrt{n} \quad \text { for } n=0,1,2, \ldots,
$$

where the supremum is over all integers $s \geq 1$, all matrix norms $\|\cdot\|$ on $\mathbb{C}^{s, s}$ and all $B \in \mathbb{C}^{s, s}$ satisfying the resolvent condition (1.2) with $M_{1}=1$.

According to the following theorem the stability estimate (2.8) can still be improved for the case of some special matrix norms.

TheOREM 2.6. Let $s \geq 1, Q \in \mathbb{C}^{s, s}$ invertible, and $p=1,2$ or $\infty$. Let the norm $\|\cdot\|$ on $\mathbb{C}^{s, s}$ be defined by $\|A\|=\left\|Q A Q^{-1}\right\|_{p}\left(\right.$ for all $\left.A \in \mathbb{C}^{s, s}\right)$. Then (1.2) with $M_{1}=1$ implies (1.1) with $M_{0}=1$ (if $p=1$ or $\infty$ ) or $M_{0}=2$ (if $p=2$ ).

Proof. Since the result for general invertible $Q$ easily follows from the result for $Q=I$, it is sufficient to consider the latter case only.

For $p=\infty$ the proof follows e.g. from [16, Theorem 2.1 and Subsection 3.1.1].

For $p=1$ the proof follows from the result for $p=\infty$ and the fact that $\|A\|_{1}=\left\|A^{\mathrm{T}}\right\|_{\infty}$ for all $A \in \mathbb{C}^{s, s}$.

For $p=2$ the value $M_{0}=2$ was stated e.g. in [31, Section 5]. The proof runs as follows. It can be seen by a straightforward calculation (or directly e.g. from the material in [2] or [16]) that the classical numerical range $\left\{\langle B x, x\rangle: x \in \mathbb{C}^{s}\right.$ with $\langle x, x\rangle=1\}$, where $\langle\cdot, \cdot\rangle$ is the usual inner product, is contained in the unit disk $D$. Therefore, by Berger's inequality, one arrives at (1.1) with $M_{0}=2$ (see e.g. [29], [32, p. 89] or [2, Section 1]). 
2.4. About the best stability estimates for fixed $M_{1}>1$. Theorem 2.1 shows that if the resolvent condition (1.2) is satisfied with fixed $M_{1}$, then $\left\|B^{n}\right\|$ can grow at most linearly with $n$ or $s$. Corollary 2.3 reveals that the corresponding upper bound is sharp - if we allow $M_{1}$ to become arbitrarily large.

For the special case $M_{1}=1$, however, this linear growth with $n$ or $s$ is too pessimistic, as can be seen from Theorems 2.4 and 2.6 in the previous subsection.

Also for other fixed values $M_{1}>1$ the question arises whether the upper bound (2.4) can be improved. In the following we present two (negative) results, showing that growth with $\sqrt{n}$ or (almost) $\sqrt{\log s}$ and $\sqrt{\log n}$ may occur. The proofs of these results are related to arguments in [21] and [22], respectively. that

TheOREM 2.7. Let $p=1$ or $p=\infty$. Then there exist $C>0$ and $M>1$ such

$$
\sup _{s, B}\left\|B^{n}\right\|_{p} \geq C \sqrt{n} \quad \text { for } n=0,1,2, \ldots,
$$

where the supremum is over all integers $s \geq 1$ and all matrices $B \in \mathbb{C}^{s, s}$ satisfying the resolvent condition (1.2) with $M_{1}=M$ and $\|\cdot\|=\|\cdot\|_{p}$.

Theorem 2.8. Let $M>\pi+1$ and $\beta \in\left(0, \frac{1}{2}\right)$ be given. Then there exist a constant $C>0$ and matrices $B_{s} \in \mathbb{C}^{s, s}$ for $s=2,4,6, \ldots$, such that all $B_{s}$ satisfy (1.2) with $M_{1}=M,\|\cdot\|=\|\cdot\|_{2}$, and

$$
\left\|\left(B_{s}\right)^{s / 2}\right\|_{2} \geq C(\log s)^{\beta} \text {. }
$$

\section{Stability estimates under resolvent conditions with respect to general regions $V$}

3.1. Failing of the eigenvalue condition. Let an initial-boundary value problem be given for a linear partial differential equation with variable coefficients in the differential operator which depend on the space variable $x$ but not on the time variable $t$. Applying the method of semi-discretization, where discretization is applied to the space variable $x$ only, one generally arrives at an initial value problem for a large system of ordinary differential equations of type

$$
\left\{\begin{array}{l}
U^{\prime}(t)=A U(t)+b(t) \quad(t \geq 0), \\
U(0)=u_{0} .
\end{array}\right.
$$

Here the (time-independent) matrix $A \in \mathbb{C}^{s, s}$, the inhomogeneous term $b(t) \in \mathbb{C}^{s}$ and the initial value $u_{0} \in \mathbb{C}^{s}$ are determined by the original initial-boundary value problem and by the process of semi-discretization. The solution to the original initial-boundary value problem is then approximated by the solution $U(t) \in \mathbb{C}^{s}$ to $(3.1)$.

Many step-by-step methods for the numerical solution of ordinary differential equations, like Runge-Kutta methods or Rosenbrock methods, reduce - when applied to (3.1) — to a fully discrete numerical process of type

$$
u_{n}=\varphi(h A) u_{n-1}+b_{n} \quad \text { for } n=1,2,3, \ldots
$$


Here $\varphi$ is a rational function depending only on the underlying step-by-step method, with $\varphi(0)=\varphi^{\prime}(0)=1$, and $h=\Delta t>0$ denotes the step size. Further, the vectors $b_{n} \in \mathbb{C}^{s}$ are related to $b(t)$, and the vectors $u_{n}$, calculated successively from (3.2), are intended to approximate $U(n h)$.

For $n \geq 1$ we denote by $\widetilde{u}_{n}$ the approximations to $U(n h)$ which are obtained by the process (3.2) when the starting vector $u_{0}$ is replaced by a slightly perturbed vector $\widetilde{u}_{0}$. The process $(3.2)$ is said to be stable if a small perturbation $v_{0}=\widetilde{u}_{0}-u_{0}$ always yields errors $v_{n}=\widetilde{u}_{n}-u_{n}$ (for $n \geq 1$ ) that are also small. Therefore, the stability analysis of (3.2) amounts to bounding $v_{n}$ (for $n \geq 1$ ) suitably in terms of $v_{0}$.

Since $v_{n}=\varphi(h A)^{n} v_{0}$, we are interested in deriving upper bounds for $\left\|\varphi(h A)^{n}\right\|$, where $\|\cdot\|$ is a norm on $\mathbb{C}^{s, s}$.

Let

$$
S=\{\zeta: \zeta \in \mathbb{C} \text { and }|\varphi(\zeta)| \leq 1\}
$$

denote the so-called stability region of the step-by-step method under consideration. A well-known criterion for stability of the process (3.2) is the so-called eigenvalue condition

$$
\sigma[h A] \subset \operatorname{int}(S) .
$$

Here $\sigma[h A]$ denotes the spectrum of $h A$ and $\operatorname{int}(S)$ the interior of $S$. If (3.4) is satisfied, the spectral mapping theorem (see e.g. [4]) guarantees that $\sigma[\varphi(h A)] \subset$ $\operatorname{int}(D)$, which implies $\varphi(h A)^{n} \rightarrow 0$ as $n \rightarrow \infty$ (see e.g. [9, p. 298]). Therefore, there exists a (finite) constant $M_{0}$ such that

$$
\left\|\varphi(h A)^{n}\right\| \leq M_{0} \quad \text { for } n=0,1,2, \ldots
$$

If $\|\cdot\|$ stands for the spectral norm and the matrix $A$ is normal, one readily arrives at the value $M_{0}=1$. In this case we even have $M_{0}=1$ if (3.4) would be replaced by the weaker condition $\sigma[h A] \subset S$. However, for matrices that are not normal, the stronger condition (3.4) does not prevent $M_{0}$ from being excessively large (see e.g. [6], [10], [13], [15], [17], [27], [31], [32], [39]). In fact, (3.4) is a notoriously unreliable condition due to the fact that $M_{0}$ is not only related to the eigenvalues of $h A$ but also to the condition number of the matrix composed of the (generalized) eigenvectors of $h A$. In many cases of practical interest this condition number and the best value $M_{0}$ are so large that the process (3.2) actually is quite unstable although the eigenvalue condition is satisfied (cf. loc. cit.). In the rest of this section we adapt (3.4) to a condition that reliably predicts stability — also for nonnormal matrices and norms $\|\cdot\|$ different from the spectral norm.

3.2. Reviewing stability estimates from the literature. In the literature various stability results can be found which are essentially based on the use of resolvent conditions of type

(3.6) $\quad \zeta I-h A$ is invertible and $\left\|(\zeta I-h A)^{-1}\right\| \leq M_{1} d(\zeta, V)^{-1}$ for all complex numbers $\zeta \notin V$. 
Here, $V$ is a closed subset of the stability region $S$ (see (3.3)), $M_{1}$ is a constant, $\|\cdot\|$ denotes a norm on $\mathbb{C}^{s, s}$ and $d(\zeta, V)=\min \{|\zeta-\eta|: \eta \in V\}$ is the distance from $\zeta$ to $V$. Under additional assumptions, to be stated below, it is shown in the literature that (3.6) implies a stability estimate

$$
\left\|\varphi(h A)^{n}\right\| \leq M_{1} g(n, s) \quad \text { for } n=1,2,3, \ldots,
$$

where the function $g$ only depends on $\varphi$ and $V$ (and not on $h, A, M_{1}$ or $\|\cdot\|$ ).

In the following we list some of these stability results. We assume throughout that (3.6) is satisfied with closed $V \subset S$ and a norm $\|\cdot\|$ on $\mathbb{C}^{s, s}$. In each separate case we formulate the relevant additional assumptions and the resulting function $g$. For any $W \subset \mathbb{C}$ we denote by $\partial W$ the boundary of $W$, and write $\mathbb{C}^{-}=\{\zeta: \zeta \in \mathbb{C}$ with $\operatorname{Re} \zeta \leq 0\}$.

1. In $[17$, Lemma 3.3] the estimate (3.7) is proved with $g(n, s) \equiv \gamma n$ where $\gamma$ depends only on $\varphi$ and $V$. The additional assumptions are: $V$ is bounded and convex, and $\|\cdot\|$ is induced by a vector norm on $\mathbb{C}^{s}$.

2. In [18, Theorem 2.2] the estimate (3.7) is proved with $g(n, s) \equiv \gamma s$ where $\gamma$ depends only on $\varphi$ and $V$. The additional assumptions are: $V$ is bounded and convex, $\varphi^{\prime}(\zeta) \neq 0$ on $\partial V \cap \partial S$, and $\partial V$ lies on an algebraic curve (see also [17]).

3. In [31, Theorem 7.1] the estimate (3.7) is proved with $g(n, s) \equiv \gamma \min (n, s)$ where $\gamma$ depends only on $\varphi$. The additional assumptions are: $V=S, S$ is bounded, $\varphi^{\prime}(\zeta) \neq 0$ on $\partial S$ and $\|\cdot\|$ is a weighted spectral norm (i.e. $\|B\|=\left\|D B D^{-1}\right\|_{2}$ for all $B \in \mathbb{C}^{s, s}$, where $D$ is a positive diagonal matrix).

4. In [21, Theorem 3.1] the estimate (3.7) is proved with $g(n, s) \equiv \gamma \min (n, s)$ where $\gamma$ depends only on $\varphi$. The additional assumptions are: $V=\mathbb{C}^{-}$and $\|\cdot\|$ is induced by a vector norm on $\mathbb{C}^{s}$.

5. From the material in the important paper [3] it follows that (3.7) holds with $g(n, s) \equiv \gamma \sqrt{n}$ where $\gamma$ depends only on $\varphi$. The additional assumptions are: $V=\mathbb{C}^{-}, M_{1}=1$ and $\|\cdot\|$ is induced by a vector norm on $\mathbb{C}^{s}$.

6. For $\delta \geq 0$ the wedge $W(\delta)$ is defined by $W(\delta)=\{\zeta: \zeta=0$ or $|\arg \zeta-\pi| \leq \delta\}$. In [17, Lemma 3.1] the estimate (3.7) is proved with $g(n, s) \equiv \gamma$ where $\gamma$ depends only on $\varphi$ and $V$. The additional assumptions are: $V$ is a bounded convex subset of $W(\alpha)$, where $0 \leq \alpha<\pi / 2, V \subset \operatorname{int}(S) \cup\{0\}$ and $\|\cdot\|$ is induced by a vector norm on $\mathbb{C}^{s}$.

7. In [21, Theorem 3.5] the estimate (3.7) is proved with $g(n, s) \equiv \gamma$ where $\gamma$ depends only on $\varphi$ and $V$. The additional assumptions are: $V=W(\alpha), S \supset W(\beta)$, $0 \leq \alpha<\beta \leq \pi / 2,\|\cdot\|$ is induced by a vector norm on $\mathbb{C}^{s}$ and $|\varphi(\infty)|<1$. As remarked by the authors, this result was essentially contained in [33] (see also [3, pp. 693-694], [5, Theorem 8]).

8. Let $\varrho>0$ and $D(\varrho)=\{\zeta: \zeta \in \mathbb{C}$ and $|\zeta+\varrho| \leq \varrho\}$. In [21, Theorem 3.4] the estimate (3.7) is proved with $g(n, s) \equiv \gamma \sqrt{1+n \varrho}$ where $\gamma$ depends only on 
$\varphi$. The additional assumptions are: $V=D(\varrho), S \supset \mathbb{C}^{-}$and $\|\cdot\|$ is induced by a vector norm on $\mathbb{C}^{s}$. (The assumption $S \supset \mathbb{C}^{-}$can be relaxed, see [21].)

9. The quantity $r=\sup \{\varrho: \varrho>0$ and $D(\varrho) \subset S\}$ is called the stability radius of the step-by-step method (3.2) (see e.g. [10]). Using the material in $[17$, Subsections 2.3 and 2.4] and [10], [26] or [36] it can be seen that, for $0<r<\infty$, the estimate (3.7) holds with $g(n, s) \equiv \gamma \sqrt{n}$ where $\gamma$ only depends on $\varphi$. The additional assumptions are: $M_{1}=1,\|\cdot\|=\|\cdot\|_{\infty}$ and $V=D(r)$.

Next consider $r \in(0, \infty]$ and $0<r_{0}<r$. If (3.6) holds with $V=D\left(r_{0}\right)$, then, again under the assumptions $M_{1}=1,\|\cdot\|=\|\cdot\|_{\infty}$, the inequality (3.7) even holds with $g(n, s) \equiv \gamma$, where $\gamma$ depends only on $\varphi$ and $r_{0}$ (see loc. cit.).

10. In [3], [21] more refined estimates of type (3.7) were derived for functions $\varphi$ satisfying special conditions. E.g. from [21, Theorem 3.2] it follows that, in the situation of point 4 above, an estimate (3.7) with $g(n, s) \equiv \gamma \min \left(n^{\alpha}, s\right), \alpha<1$, is possible for functions $\varphi$ with $|\varphi(\zeta)|$ not identically 1 on the imaginary axis. We refer to loc. cit. for more details.

3.3. Various comments on stability estimates from the literature

Remark 3.1. The results 1, 2, 6 and 8 in the last subsection were proved by using integral representations of type

$$
\varphi(h A)^{n}=\frac{1}{2 \pi i} \int_{\Gamma} \varphi(\zeta)^{n}(\zeta I-h A)^{-1} d \zeta,
$$

where $\Gamma$ is a proper curve in the complex plane surrounding $V$, and by estimating the integral (see e.g. the proof of (2.3)). The results 5,7 and 10 were proved by using related, but different, integral representations for $\varphi(h A)^{n}$.

The results 3 and 4 were obtained by first proving that (3.6) implies (1.2) with $B=\varphi(h A)$ and then applying (a version of) Theorem 2.1 to this matrix $B$.

Finally, the proof of result 9 relies on an expansion of $\varphi(h A)^{n}$ in a power series

$$
\gamma_{0} I+\gamma_{1}(h A+\varrho I)+\gamma_{2}(h A+\varrho I)^{2}+\ldots
$$

with $\varrho=r$ or $r_{0}$, and on bounding the terms of the series using the fact that $\|h A+\varrho I\|_{\infty} \leq \varrho$.

R e m a rk 3.2. We note that the results 1, 3, 4, 6, 7 and 8, although formulated in loc. cit. for special norms, are valid as well for arbitrary norms $\|\cdot\|$ on $\mathbb{C}^{s, s}$. This can be seen by a straightforward adaptation of the proofs in loc. cit.

Further, it is easy to see that the result 9 is also valid for norms $\|\cdot\|$ defined by $\|B\|=\left\|Q B Q^{-1}\right\|_{p}$ (for all $B \in \mathbb{C}^{s, s}$ ), where $Q$ is an invertible matrix and $p=1$ or $\infty$.

Remark 3.3. In all of the above, the resolvent condition (3.6) occurs as a sufficient condition for stability estimates of type (3.7). Reddy and Trefethen [31, Theorem 7.1] succeeded in showing (for the weighted spectral norm, see the result 3) that (3.6) is also a necessary condition for stability. In fact, they showed - for 
any matrix $h A$ belonging to a specific family $\mathcal{F}$ defined in their paper - that, in general, strong stability (i.e., $\left\|\varphi(h A)^{n}\right\| \leq M_{0}$ for all $n \geq 0$ ) implies resolvent condition (3.6) with $V=S$ and $M_{1}=\gamma M_{0}$. Here $\gamma$ depends only on $\varphi$ and $\mathcal{F}$.

Acknowledgements. The authors wish to thank S. C. Reddy and L. N. Trefethen for many stimulating discussions on the topic of this paper.

Added in proof. The full proof of the theorems stated in Section 2 can be found in the following publication of the present authors: Linear stability analysis in the numerical solution of initial value problems, Acta Numer. (1993), 199-237.

\section{References}

[1] M. Abramowitz and I. A. Stegun, Handbook of Mathematical Functions, Dover, New York 1965.

[2] F. F. Bonsall and J. Duncan, Numerical ranges, in: Studies in Functional Analysis, R. G. Bartle (ed.), Mathematical Association of America, 1980, 1-49.

[3] Ph. Brenner and V. Thomée, On rational approximations of semigroups, SIAM J. Numer. Anal. 16 (1979), 683-694.

[4] J. B. Conway, A Course in Functional Analysis, Springer, New York 1985.

[5] M. Crouzeix, On multistep approximation of semigroups in Banach spaces, J. Comput. Appl. Math. 20 (1987), 25-35.

[6] D. F. Griffiths, I. Christie and A. R. Mitchell, Analysis of error growth for explicit difference schemes in conduction-convection problems, Internat. J. Numer. Methods Engrg. 15 (1980), 1075-1081.

[7] R. D. Grigorieff, Time discretization of semigroups by the variable two-step BDF method, in: Numerical Treatment of Differential Equations, K. Strehmel (ed.), Teubner, Leipzig 1991, 204-216.

[8] P. Henrici, Applied and Computational Complex Analysis, vol. 2, Wiley, New York 1977.

[9] R. A. Horn and C. R. Johnson, Matrix Analysis, Cambridge Univ. Press, 1990.

[10] J. F. B. M. Kraaijevanger, H. W. J. Lenferink and M. N. Spijker, Stepsize restrictions for stability in the numerical solution of ordinary and partial differential equations, J. Comput. Appl. Math. 20 (1987), 67-81.

[11] H. O. Kreiss, Über die Stabilitätsdefinition für Differenzengleichungen die partielle Differentialgleichungen approximieren, BIT 2 (1962), 153-181.

[12] - Well posed hyperbolic initial boundary value problems and stable difference approximations, in: Proc. Third Internat. Conf. on Hyperbolic Problems, Uppsala, Sweden, 1990.

[13] H. O. Kreiss and L. Wu, On the stability definition of difference approximations for the initial boundary value problem, Appl. Numer. Math. 12 (1993), 213-227.

[14] G. I. Laptev, Conditions for the uniform well-posedness of the Cauchy problem for systems of equations, Soviet Math. Dokl. 16 (1975), 65-69.

[15] H. W. J. Lenferink and M. N. Spijker, The relevance of stability regions in the numerical solution of initial value problems, in: Numerical Treatment of Differential Equations, K. Strehmel (ed.), Teubner, Leipzig 1988, 95-103.

[16] - , - A generalization of the numerical range of a matrix, Linear Algebra Appl. 140 (1990), 251-266.

[17] - - - On the use of stability regions in the numerical analysis of initial value problems, Math. Comp. 57 (1991), 221-237. 
[18] H. W. J. Lenferink and M. N. Spijker, On a generalization of the resolvent condition in the Kreiss matrix theorem, ibid., 211-220.

[19] R. J. LeVeque and L. N. Trefethen, On the resolvent condition in the Kreiss matrix theorem, BIT 24 (1984), 584-591.

[20] Ch. Lubich, On the convergence of multistep methods for nonlinear stiff differential equations, Numer. Math. 58 (1991), 839-853.

[21] Ch. Lubich and O. Nevanlinna, On resolvent conditions and stability estimates, BIT 31 (1991), 293-313.

[22] C. A. McCarthy and J. Schwartz, On the norm of a finite boolean algebra of projections, and applications to theorems of Kreiss and Morton, Comm. Pure Appl. Math. 18 (1965), 191-201.

[23] J. Miller, On the resolvent of a linear operator associated with a well-posed Cauchy problem, Math. Comp. 22 (1968), 541-548.

[24] J. Miller and G. Strang, Matrix theorems for partial differential and difference equations, Math. Scand. 18 (1966), 113-123.

[25] K. W. Morton, On a matrix theorem due to H. O. Kreiss, Comm. Pure Appl. Math. 17 (1964), 375-379.

[26] O. Nevanlinna, Remarks on time discretization of contraction semigroups, Helsinki Univ. Techn., Inst. Math., report HTKK-MAT-A225 (1984).

[27] S. V. Parter, Stability, convergence, and pseudo-stability of finite-difference equations for an over-determined problem, Numer. Math. 4 (1962), 277-292.

[28] A. Pazy, Semigroups of Linear Operators and Applications to Partial Differential Equations, Springer, New York 1983

[29] C. Pearcy, An elementary proof of the power inequality for the numerical radius, Michigan Math. J. 13 (1966), 289-291.

[30] S. C. Reddy and L. N. Trefethen, Lax-stability of fully discrete spectral methods via stability regions and pseudo-eigenvalues, Comput. Methods Appl. Mech. Engrg. 80 (1990), 147-164.

[31] —, - Stability of the method of lines, Numer. Math. 62 (1992), 235-267.

[32] R. D. Richtmyer and K. W. Morton, Difference Methods for Initial-Value Problems, 2nd ed., Wiley, New York 1967.

[33] M.-N. Le Roux, Semidiscretization in time for parabolic problems, Math. Comp. 33 (1979), 919-931.

[34] W. Rudin, Functional Analysis, McGraw-Hill, New York 1973.

[35] J. C. Smith, An inequality for rational functions, Amer. Math. Monthly 92 (1985), 740-741.

[36] M. N. Spijker, Stepsize restrictions for stability of one-step methods in the numerical solution of initial value problems, Math. Comp. 45 (1985), 377-392.

[37] - On a conjecture by LeVeque and Trefethen related to the Kreiss matrix theorem, BIT 31 (1991), 551-555.

[38] E. Tadmor, The equivalence of $L_{2}$-stability, the resolvent condition and strict $H$-stability, Linear Algebra Appl. 41 (1981), 151-159.

[39] L. N. Trefethen, Non-normal matrices and pseudospectra, in preparation.

[40] E. Wegert and L. N. Trefethen, From the Buffon needle problem to the Kreiss matrix theorem, Amer. Math. Monthly, to appear. 